\title{
Simulation study of wormhole formation and propagation during matrix acidizing of carbonate reservoirs using a novel in-situ generated hydrochloric acid
}

\author{
Safa Kiani ${ }^{1}$, Saeed Jafari ${ }^{2 \oplus *}$, Saeid Norouzi Apourvari ${ }^{1}$, Hossein Mehrjoo ${ }^{1}$ \\ ${ }^{1}$ Department of Petroleum Engineering, Shahid Bahonar University of Kerman, Kerman 009834, Iran \\ ${ }^{2}$ Department of Mechanical Engineering, Shahid Bahonar University of Kerman, Kerman 009834, Iran
}

Keywords:

Hydrochloric acid

wormhole propagation

two-scale model

finite-volume method

acidizing

Cited as:

Kiani, S., Jafari, S., Apourvari, S. N., Mehrjoo, H. Simulation study of wormhole formation and propagation during matrix acidizing of carbonate reservoirs using a novel in-situ generated hydrochloric acid. Advances in Geo-Energy Research, 2021, 5(1): 64-74, doi: 10.46690/ager.2021.01.07

\begin{abstract}
:
Optimum acid selection based on reservoir condition is one of the key elements in achieving a successful treatment in carbonate matrix acidizing. High reaction and corrosion rate of regular hydrochloric acid especially in tight reservoirs with high temperatures is considered as a limitation of this acid. One alternative in a high-temperature reservoir is to use insitu generated hydrochloric. The objective of this study is to simulate acidizing treatment using both regular hydrochloric and in-situ generated hydrochloric acid. A continuum twoscale model for linear and radial flow geometry was used to simulate dissolution pattern. A finite-volume method with an implicit backward Euler scheme was used to solve the governing equations. The results of the simulation in a linear system showed that, due to low face dissolution, the in-situ generated hydrochloric at low injection rates could create longer wormholes with less pore volume to breakthrough. The results also proved that using the in-situ generated hydrochloric acid could lead to less face dissolution and deeper penetration of acid into the reservoir. This acidizing system is very suitable in situations where a low injection rate is needed and regular hydrochloric cannot be used due to higher pore volume to breakthrough and high corrosion rate.
\end{abstract}

\section{Introduction}

More than half of the reserves in the Middle East are located in carbonate reservoirs. Matrix acidizing is one of the best techniques to stimulate these reservoirs. If the treatment is successful, conductive channels, known as wormholes, will be formed that could bypass the damaged zone. Wormholes make the connection between formation and wellbore. Therefore, hydrocarbons can flow to the wellbore through new created flow channels. In carbonate reservoir, reactive flow dissolves the rock and wormhole will be created, while in sandstone, reactive fluid dissolves the damage that existed inside the pore and wormholes are not formed in this type of reservoir. Creation of wormholes in carbonate reservoir that penetrate beyond the damaged zone, can result in a negative skin in this type of formation (Glasbergen et al., 2009). The lowest skin factors will be achieved when the acidizing treatment is conducted at optimum injection rate, and consequently the longest wormholes are created (Akanni and Nasr-El-Din, 2015). Decreasing the skin factor depends on the dissolution pattern which influences the invasion of the acid into the reservoir (Fredd, 2000). The highest acid penetration occurs when the dominant wormhole is formed and this is led to the highest reduction in the skin factor. For this purpose, fluid type, injection flow rate, and fluid volume must be investigated (Glasbergen et al., 2009). The importance of the injection rate on wormhole formation has also been pointed out by McDuff et al. (2010). The optimum injection rate of acid was determined and it was shown that at a specific temperature, the acid injection rate is an important parameter to form wormholes. As highlighted by Akanni and Nasr-ElDin (2015), an optimum acid injection rate in matrix acidizing treatments is very important to achieve deep acid penetration. Suitable acid selection for high-temperature tight reservoirs,

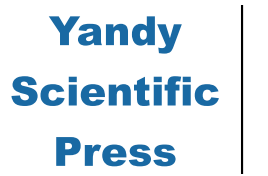

* Corresponding author.

E-mail address: safa.k875@yahoo.com (S. Kiani); jafari@uk.ac.ir (S. Jafari); saeednowroozi@gmail.com (S. N. Apourvari);

mehrjoo.hmehrjoo.hossein@gmail.com (H. Mehrjoo).

2207-9963 (c) The Author(s) 2021.

Received November 29, 2020; revised January 5, 2021; accepted January 5, 2021; available online January 8, 2021. 
in which a high acid injection rate is a limitation, could be challenging In situations where a high injection rate is a limitation, the acid would be injected at a lower injection rate than the optimal rate. The low injection rate of hydrochloric $(\mathrm{HCl})$ causes face dissolution and shallow penetration of the acid. Weak and retarded acids are some alternatives to $\mathrm{HCl}$ at high temperatures reservoirs and reservoirs in which high injection rates are not possible. Due to the lower reaction rate and lower corrosion rates, organic acids could be used at deep and high-temperature reservoirs (Chang and Nasr-El-Din, 2008). However, there are some drawbacks associated with these acids such as the availability of less $\mathrm{H}^{+}$by increasing the temperature. This requires more acid and as a result, the cost of the acidizing treatments increases. Moreover, reaction products, such as calcium formate and calcium acetate, have low solubility compared to calcium chloride that may lead to precipitation plugging (Aldakkan et al., 2018). Organic acids also need extra additive to improve the effectiveness of the treatment (Shuchart and Gdanski, 1996). Emulsified acids are classified as retarded acids that compared to conventional acids have a lower diffusion coefficient and consequently a lower reaction rate. The relative stability of these acids could be influenced by temperature, the volume fraction of acid, the mixing method, droplet size and distribution of droplets and shear rate (Mason et al., 1996; Al-Mutairi et al., 2007, 2009). Long-term stability at high temperatures is a current challenge of the emulsion systems (Cairns et al., 2016). Another alternative to regular $\mathrm{HCl}$ acid is to use chelating agents that not only have less reaction and corrosion rates, but also high dissolving power, less tendency to form sludge and precipitation of asphaltene, and more compatibility with the environment (Mahmoud and Nasr-El-Din, 2015; Nasr-ElDin et al., 2015). Compared to $\mathrm{HCl}$, these acids are more expensive, and their thermal stability is also a concern at high temperatures (Sokhanvarian et al., 2016). A mixture of organic and $\mathrm{HCl}$ acids was proposed by Kankaria et al. (2017). They concluded that using this system leads to longer and more effective wormholes compared to either $\mathrm{HCl}$ or organic acids. Moreover, the treatment cost decreases as a lower pore volume (PV) of acid are needed. Arslan et al. (2017) introduced an in-situ generated $\mathrm{HCl}$ as an alternative to regular $\mathrm{HCl}$. They carried out dissolution experiments using a rotating disk apparatus (RDA) and emphasized that the reaction rate of regular $\mathrm{HCl}$ is nearly twice as in-situ generated $\mathrm{HCl}$ at $100^{\circ} \mathrm{F}$. The diffusion coefficient of in-situ generated $\mathrm{HCl}$ at $150{ }^{\circ} \mathrm{F}$ was smaller than that of regular $\mathrm{HCl}$ at $100{ }^{\circ} \mathrm{F}$. Activation energy of introduced acid was calculated and it was 1.7 and 2.9 higher than acetic acid and regular $\mathrm{HCl}$, respectively. Sokhanvarian et al. (2017) conducted core flood experiments on dolomite and limestone samples using the same acid system. They observed that the needed pore volume to breakthrough (PVBT) of acid for in-situ generated $\mathrm{HCl}$ was less than that for regular $\mathrm{HCl}$ for both dolomite and limestone samples. Another shortcoming of regular $\mathrm{HCl}$ that was addressed using in-situ generated $\mathrm{HCl}$ was the high corrosion rate. The corrosion rate of in-situ generated $\mathrm{HCl}$ was significantly less compared to regular $\mathrm{HCl}$ at $300{ }^{\circ} \mathrm{F}$ (Sokhanvarian et al., 2017). Due to encouraging results obtained for in-situ generated $\mathrm{HCl}$ acid, the performance of this acid system is simulated in our study.

The analysis of acidizing treatment in experimental settings at the lab scale or even larger can be time consuming and expensive. Moreover, it cannot be representative of field treatments. Mathematical modeling and simulations could be very effective in designing successful acidizing treatments at larger scales (Liu et al., 1997). There are different models for carbonate matrix acidizing treatments such as capillary tube approach, semi-empirical approach, Peclet number approach, transition pore theory, network models, two-scale model (TSM), transition pore theory, and Damkhohler number approach. The advantages and disadvantages of these models were reviewed by Akanni and Nasr-El-Din (2015). The TSM with considering the reaction and transport of acid at both pore and core scale is known as the best model for predicting the formation and propagation of wormholes (Akanni and NasrEl-Din, 2015). Furthermore, this model can also be used to determine the optimum injection rate at the core scale, but its application at the field scale is computationally intensive (Akanni and Nasr-El-Din, 2015; Liu et al., 2018). Liu et al. (1997) developed a continuum model for sandstone reservoirs, which was also capable to predict the wormhole formation in carbonate reservoirs. A Darcy-scale model was presented by Golfier et al. (2002) for predicting dissolution patterns in carbonate reservoirs. These models are only valid either for reaction rate-limited or mass transfer limited situations. A twoscale continuous (TSC) model was first established by Panga et al. $(2004,2005)$ to investigate initiation and propagation of wormhole in carbonates. The effect of reaction rate regime, wormhole density, and dimension were also studied in this model (Panga et al., 2004). This model was later extended by Cohen et al (2007) for radial flow to investigate the effect of flow geometry on the PVBT curve. They showed that the optimum injection rete in radial flow is higher than that of linear flow. Porosity distribution was taken from X-ray CT and a TSM was utilized by Ali and Nasr-El-Din (2019) to simulate wormhole propagation under field conditions. They could not find any direct relation between acid propagation under field conditions and experimental results. Later, a largescale radial TSC model was developed by Ali and Nasr-El-Din (2020) to simulate acidizing treatment under field conditions and Recently, a 3D model for fractured carbonate rocks was developed by Yuan and Guan (2020). Instead of Darcy and Navier-Stokes equations, the Stokes-Brinkman equation was used and the coupling effects of fluid flow and reactive transport on dissolution patterns was studied (Yuan and Guan, 2020).

Based on the development of new acidizing systems and multi-variable models, the simulation of new acidizing systems on a bigger scale is useful. The objective of this study is to simulate the matrix acidizing process in carbonate rock and particularly to compare the dissolution pattern of regular $\mathrm{HCl}$ with newly developed in-situ generated $\mathrm{HCl}$ by Arslan et al. (2017). Both radial and linear systems are considered in simulations. The simulation of acidizing treatment using insitu generated acid in radial geometry has never done before. A two-scale model is built based on the Panga model (Panga et al., 2004), and similar to regular $\mathrm{HCl}$, a linear reaction kinetic 
between the in-situ generated $\mathrm{HCl}$ and calcite is considered. The effect of temperature on dissolution patterns of these two systems is also investigated.

\section{Mathematical model}

\subsection{Linear model}

In this study two dimension linear flow is simulated using a TSC. The following is a brief description of this model. Detailed description could be found in Panga et al. (2005).

\subsubsection{Darcy-scale model}

The fluid flow can be described by Darcy's law equation:

$$
U=\frac{-K}{\mu} \cdot \nabla P
$$

In above equation, $U(u, v, w)$ shows the Darcy velocity vector, $\mu$ and $P$ are viscosity and pressure, respectively. $K$ shows permeability tensor. The effect of porosity change on the flow field during dissolution can be explained by the continuity equation, Eq. (2):

$$
\frac{\partial \varepsilon}{\partial t}+\nabla \cdot U=0
$$

Because of the reactive transport of the acid inside the porous media, the concentration of the acid changes with time and local coordinates. Therefore, the mass balance equation for acid is as follows:

$$
\frac{\partial\left(\varepsilon C_{f}\right)}{\partial t}+\nabla \cdot\left(U C_{f}\right)=\nabla \cdot\left(\varepsilon D_{e} \cdot \nabla C_{f}\right)-K_{c} a_{v}\left(C_{f}-C_{s}\right)
$$

where $\varepsilon$ denotes the porosity of the medium, the cup-mixing concentration of the acid in the fluid phase is shown by $C_{f}$ and $C_{s}$ shows the concentration of acid at the solid-fluid interface. $D_{e}$ shows the effective dispersion tensors of acid, $K_{c}$ denotes the local mass transfer coefficient, $a_{v}$ represents the interfacial area per unit volume of the medium. The reaction kinetics in Eq. (4) balance the quantity of acid transported from the fluid phase to the solid surface to the quantity reacted at the surface.

$$
K_{c}\left(C_{f}-C_{S}\right)=R\left(C_{S}\right)
$$

The porosity-evolution equation, obtained from the equivalence between the amount of acid consumed and the rock dissolved is:

$$
\frac{\partial \varepsilon}{\partial t}=\frac{R\left(C_{s}\right) \alpha a_{v}}{\rho_{s}}
$$

where $R\left(C_{s}\right)$ represents the reaction kinetics, $\alpha$ shows the dissolving power of the acid and $\rho_{s}$ denotes the density of the rock. For a first-order reaction:

$$
R\left(C_{s}\right)=K_{s} C_{s}
$$

Here $K_{s}$ is the dissolution-rate constant, and then Eq. (4) is modified to

$$
C_{s}=\frac{C_{f}}{1+\frac{K_{s}}{K_{c}}}
$$

\subsection{Dimensionless form of two-scale model}

Eqs. (1)-(5) are converted to dimensionless forms using dimensionless parameters and dimensionless numbers defined below.

$$
\begin{gathered}
x^{*}=\frac{x}{L}, y^{*}=\frac{y}{H}, u^{*}=\frac{u}{u_{0}}, v^{*}=\frac{v}{u_{0}}, t^{*}=\frac{t}{L / u_{0}} \\
r=\frac{r_{p}}{r_{0}}, A_{v}=\frac{a_{v}}{a_{0}}, k^{*}=\frac{k}{k_{0}}, c_{f}=\frac{C_{f}}{C_{0}}, c_{s}=\frac{C_{s}}{C_{0}} \\
p=\frac{P-P_{e}}{\frac{\mu u_{0} L}{k_{0}}}, \varphi^{2}=\frac{2 K_{s} r_{0}}{D_{m}}, \eta=\frac{2 r_{0}}{L}, \alpha_{0}=\frac{H}{L} \\
D a=\frac{K_{s} a_{0} L}{u_{0}}, N_{a c}=\frac{\alpha C_{0}}{\rho_{s}}, P e_{L}=\frac{u_{0}}{D_{m}}, \Phi^{2}=\frac{K_{s} a_{0} L^{2}}{D_{m}}
\end{gathered}
$$

In above relations, $L$ and $H$ show the characteristic length scale in $\mathrm{x}$-direction and height of the area, respectively. $u_{0}$ represents inlet velocity. $a_{0}, r_{0}$ and $r_{p}$ indicate initial interfacial area, initial and average pore radius, respectively. Initial permeability is represented by $k_{0} . C_{0}$ is inlet concentration of acid and $P_{e}$ shows outlet pressure. The effective molecular diffusivity is shown by $D_{m}$. Four dimensionless numbers, core scale or macro Damkohler number, acid capacity number, the axial Peclet number and macroscopic Thiele modulus are defined by $D a, P e_{L}, N_{a c}$ and $\Phi^{2}$, respectively. It is worth mentioning that $\varphi^{2}$ in Eq. (10) shows pore-scale Thiele modulus. As shown in Eq. (11), macroscopic Thiele modulus is to reach a parameter independent of the injection velocity. The ratio of convective time to reaction time based on the length scale of the core is called $D a$. The ratio of the volume of solid dissolved per unit volume of the acid is known as Nac. Peclet number, $P e_{L}$, is defined as the ratio of axial diffusion time to convection time. The dimensionless forms of Eqs. (1)(5) are given below.

$$
\begin{gathered}
\left(u^{*}, v^{*}\right)=\left(-k^{*} \frac{\partial p}{\partial x^{*}},-k^{*} \frac{\partial p}{\partial y^{*}}\right) \\
\frac{\partial \varepsilon}{\partial t^{*}}+\frac{\partial u^{*}}{\partial x^{*}}+\frac{\partial v^{*}}{\partial y^{*}}=0
\end{gathered}
$$

$$
\begin{aligned}
& \frac{\partial\left(\varepsilon c_{f}\right)}{\partial t}+\frac{\partial\left(u^{*} c_{f}\right)}{\partial x^{*}}+\frac{\partial\left(v^{*} c_{f}\right)}{\partial y^{*}}=-\frac{D a A_{v} c_{f}}{1+\frac{\varphi^{2} r}{S h}}+A+B \\
& A=\frac{\partial}{\partial x^{*}}\left(\frac{\alpha_{o s} D a}{\Phi^{2}}+\lambda_{x}|U| r \eta \frac{\partial c_{f}}{\partial y^{*}}\right) \\
& B=\frac{\partial}{\partial y^{*}}\left(\frac{\alpha_{o s} D a}{\Phi^{2}}+\lambda_{T}|U| r \eta \frac{\partial c_{f}}{\partial y^{*}}\right)
\end{aligned}
$$




$$
\frac{\partial \varepsilon}{\partial t}=\frac{D a A_{\nu} c_{f} N_{a c}}{1+\frac{\varphi^{2} r}{S h}}
$$

\subsection{Pore-scale equations}

Structure-property relationships are applied by the porescale model to relate the evolution in permeability to the local porosity, pore radius, and interfacial surface area of the medium once dissolution happens. Eqs. (16)-(18) presented relations between the above-mentioned variables:

$$
\begin{gathered}
\frac{k}{k_{0}}=\frac{\varepsilon}{\varepsilon_{0}}\left(\frac{\varepsilon\left(1-\varepsilon_{0}\right)}{\varepsilon_{0}(1-\varepsilon)}\right)^{2 \beta} \\
\frac{r_{p}}{r_{0}}=\sqrt{\frac{k \varepsilon_{0}}{k_{0} \varepsilon}} \\
\frac{a_{v}}{a_{0}}=\sqrt{\frac{\varepsilon r_{0}}{\varepsilon_{0} r_{p}}}
\end{gathered}
$$

where $\beta$ is a constant which could be influenced by the structure of the porous media. $\varepsilon_{0}, k_{0}$ and $r_{0}$ are the initial values of porosity, permeability and average pore radius. The local mass-transfer and effective-dispersion coefficient of acid species are obtained by use of the following correlations Panga et al. (2005):

$$
\begin{gathered}
S h=\frac{2 K_{c} r_{p}}{D_{m}}=S h_{\infty}+0.7 R_{e}{ }^{\frac{1}{2}} S c^{\frac{1}{3}} \\
D_{e x}=\alpha_{o s} D_{m}+\frac{2 \lambda_{x}|U| r_{p}}{\varepsilon} \\
D_{e T}=\alpha_{o s} D_{m}+\frac{2 \lambda_{T}|U| r_{p}}{\varepsilon}
\end{gathered}
$$

In the above equation, $S h$ is the dimensionless mass transfer coefficient and also known as the Sherwood number. $S h_{\infty}$ is the asymptotic Sherwood number. Reynolds number, $R_{e}$, is defined as $R_{e}=2|U| r_{p} / v$. Schmidt number, $S c$, is defined as $S c=v / D_{m}$, where $v$ is kinematic viscosity and $D_{m}$ is the molecular diffusivity of the acid, $\alpha_{o s}$ is a constant that can be determined based on the structure of the porous media. $D_{e x}$ shows the longitudinal-dispersion coefficient and $D_{e T}$ shows the transverse-dispersion coefficient. $\lambda_{x}$ and $\lambda_{T}$ are structure dependent constants. $\lambda_{x}$ is approximately 0.5 and $\lambda_{T}$ is approximately 0.1 for a packed bed of spheres. The non-dimensional of longitudinal-dispersion coefficient and transverse-dispersion coefficient can be written as:

$$
\begin{gathered}
D_{e x}=\frac{\alpha_{o s} D a}{\Phi^{2}}+\lambda_{x}|U| r \eta \\
D_{e T}=\frac{\alpha_{o s} D a}{\Phi^{2}}+\lambda_{T}|U| r \eta
\end{gathered}
$$

\section{Numerical Simulation}

The numerical solution of Eqs. (2)-(5) is accomplished by finite-volume method with an implicit backward Euler scheme. First, Eq. (2), continuity equation, is solved to obtain the pressure and velocity distribution in the porous medium. Second, the transport equation (Eq. (3)) is solved, followed by the porosity-evolution equation (Eq. (5)) to calculate the change in porosity. The pore-scale model is then implemented to renew the pore characteristics at each time step while the breakthrough condition is being checked. This continues until a fluid breakthrough is estimated to occur at the outlet of the domain. In our study, the breakthrough condition is reached once the overall pressure decrease across the domain is reduced 100 times compared to the initial pressure drop. The workflow of the simulation process is denoted in Fig. 1.

The domain was discretized by using square meshes with fixed size at $300 \times 120$ (300 cells in the $\mathrm{x}$-direction and 120 cells in the y-direction). In other words, an aspect ratio of 0.4 was used in this work.

\subsection{Initial and boundary conditions}

In order to denote heterogeneity in the medium, an accidental fluctuation in the initial porosity was introduced. This accidental fluctuation is dispersed in the interval $\left[-\Delta \varepsilon_{0}, \Delta \varepsilon_{0}\right]$ and then added to the average value of initial porosity as follow:

$$
\varepsilon=\varepsilon_{0}+\xi \quad \text { At } t=0,
$$

where $\xi$ varies randomly from $-\Delta \varepsilon$ to $\Delta \varepsilon$. Velocity field and concentration in field are zero.

Boundary conditions for Eq. (2) are a constant injection velocity at the inlet, depending on the input for a specific simulation run, and constant pressure at the outlet of the domain. For Eq. (3) we use the Danckwerts condition (Danckwerts, 1953) at the inlet and at the core outlet, the acid flux is set to zero. No flow boundary condition was assumed on the lateral sides of the core.

\subsection{Base case}

To simulate the matrix acidizing of $15 \mathrm{wt} \%$ regular $\mathrm{HCl}$ on calcite, the base case is modeled. Table 1 shows the values of the parameters that used in simulating the base case. These values were gained from experimental (Fredd and Fogler, 1998) and simulation study (Kalia and Balakotaiah, 2009), as shown in Table 1. It is worth mentioning that numerical experiments were carried out at $73{ }^{\circ} \mathrm{F}$.

\section{Results and discussion}

\subsection{PVBT curves}

Injected PV as a function of rate or inverse $D a$ could be represented by PVBT curve and could be used to define the minimum injected PVBT. $D a$, which indicates the dimensionless form of the injection rate, is defined as the ratio of convection time to the reaction time of the Darcy scale (Panga et al., 2005). 


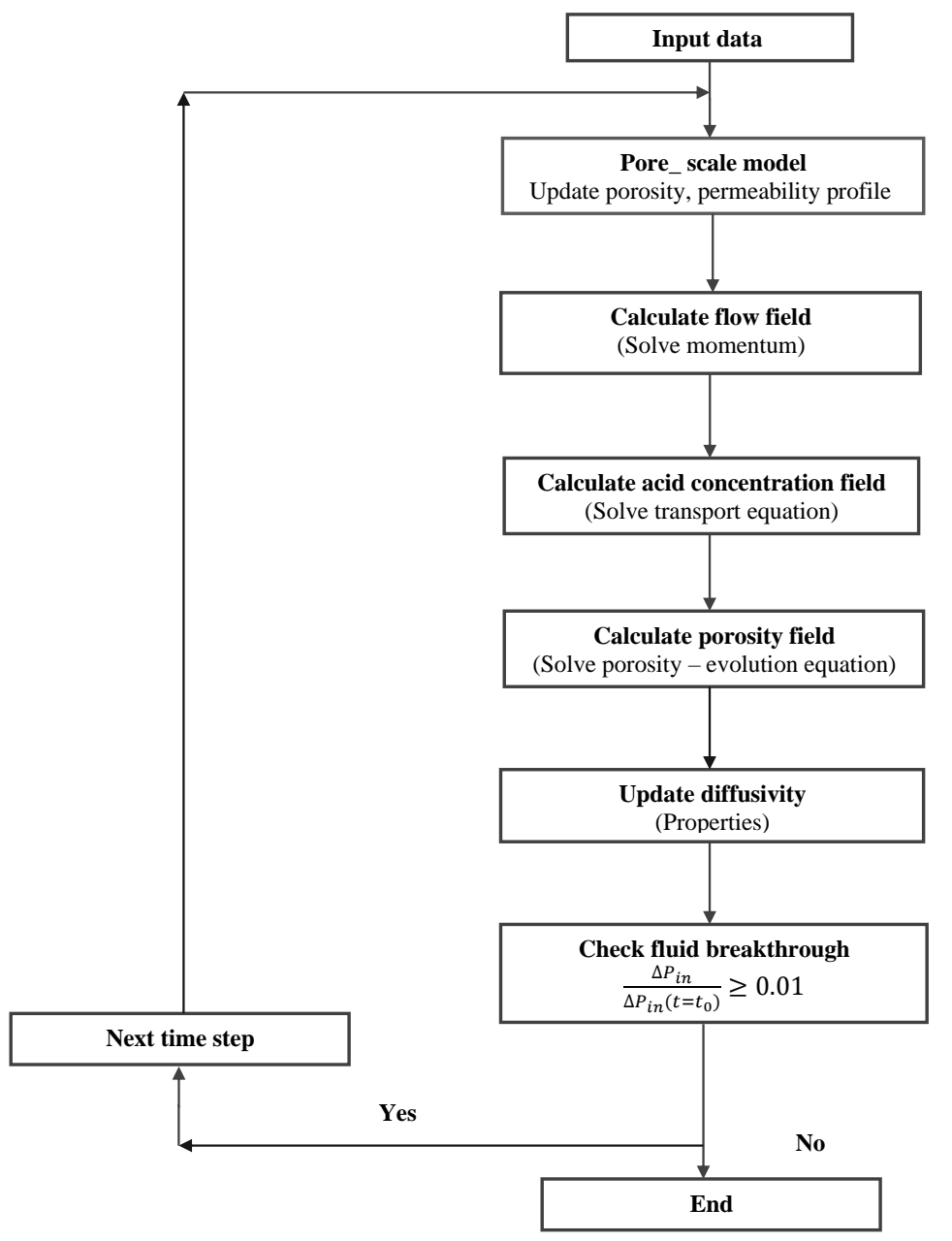

Fig. 1. The flow chart of the numerical model.

Table 1. Input parameters and their values used in numerical simulations of the base case model (Fredd and Fogler, 1998; Kalia and Balakotaiah, 2009).

\begin{tabular}{ll}
\hline Input parameter & Value \\
\hline Acid diffusivity $\left(D_{m}\right)$ & $3.6 \mathrm{e}-5(\mathrm{~cm} / \mathrm{s})$ \\
Acid dissolving power $(\alpha)$ & 1.37 \\
Acid viscosity $(\mu)$ & $1(\mathrm{cp})$ \\
Asymptotic Sherwood number $\left(S h_{\infty}\right)$ & 3 \\
Average porosity $\left(\varepsilon_{0}\right)$ & 0.2 \\
Constant in dispersion correlation $\left(\alpha_{o s}\right)$ & 0.5 \\
Constant in axial-dispersion correlation $\left(\lambda_{x}\right)$ & 0.5 \\
Constant in transverse-dispersion correlation $\left(\lambda_{T}\right)$ & 0.1 \\
Initial average permeability $\left(k_{0}\right)$ & $15(\mathrm{md})$ \\
Initial interface area per unit volume $\left(a_{0}\right)$ & $50\left(\mathrm{~cm}{ }^{-1}\right)$ \\
Initial mean pore size $\left(r_{0}\right)$ & $5.2 \mathrm{e}-6(\mathrm{~cm})$ \\
Pore- structure-relation constant $(\beta)$ & 1 \\
Porosity-heterogeneity magnitude $\left(\Delta \varepsilon_{0}\right)$ & \pm 0.15 \\
Surface-dissolution reaction-rate constant $\left(K_{s}\right)$ & $0.2(\mathrm{~cm} / \mathrm{s})$ \\
\hline
\end{tabular}

The acidizing process for $15 \mathrm{wt} \%$ regular $\mathrm{HCl}$ is first simulated on calcite. Similar to simulation study of Kalia and Balakotaiah (2009) in our simulation study for 2D linear flow model, domain is rectangular with dimension of $4 \times 10 \mathrm{~cm}$. To ensure the accuracy of the model's performance, a comparison was made between the results of the model with that of Kalia and Balakotaiah. Fig. 2 shows the PVBT curve obtained from our simulations and its comparison with the results of the Kalia and Balakotaiah (2009).

A dimensionless $D a$ determines the effects of both the acid reaction rate and the fluid velocity. The shape of dissolution patterns is determined by the relative magnitudes of reaction, dispersion, and convection in the acid transport Eq. (3). Different dissolution patterns are resulted of alteration of the magnitudes of the transport and reaction mechanisms when the $D a$ in Figs. 3(a)3-(f) decreases. For example, at high $D a$ (low injection rates), the main phenomena are the chemical reaction and acid dispersion, Therefore the acid can be consumed at the beginning of the domain before it can enter into the domain, resulting in the formation of face dissolution (Fig. 3(a)). With decreasing $D a$, convection controls the acid transport and patterns with favored dissolution, known as conical wormholes, are formed (Fig. 3(b)). Although the convection time scale is 


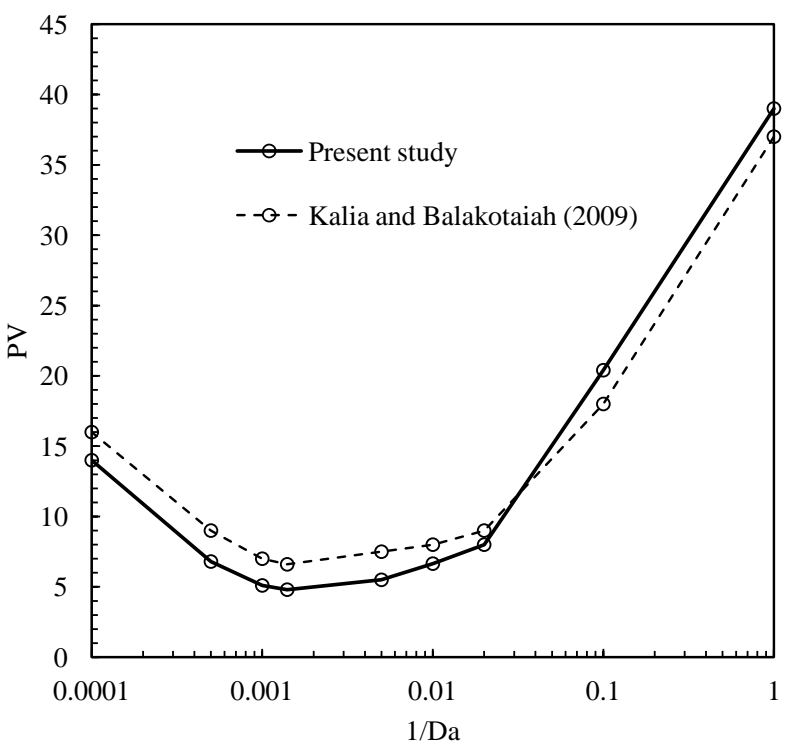

Fig. 2. The acid efficiency curve. Showing PVBT as a function of injection rate $(1 / D a)$ for the $2 \mathrm{D}$ numerical simulation with linear flow.

greater than that in the compact dissolution regime, it is not comparable to the dispersion and reaction scales. Dispersion, reaction and convection time scales are of comparable magnitude giving rise to wormholes when $D a$ is further decreased (Fig. 3(c)). The required amount of acid to breakthrough reduces with decreasing $D a$ untill a certain value $D a$, opt is reached. Increasing the velocity over and above the optimal amount causes the acid to spend over on the walls of the wormhole, resulting in the formation of a branched structure, and eventually, injection of acid at very high rates leads to a uniform dissolution formation (Figs. 3(d)-3(f)).

\subsection{Effect of temperature}

In order to study the effect on temperature on PVBT and optimum rate, its effect on the dissolution rate constant is involved in the model. The relationship between the rate of reaction and temperature for a first-order reaction has been given by Kalia and Glasbergen (2009).

$$
R\left(C_{s}, T_{2}\right)=K_{s}\left(T_{2}\right) C_{s}=K_{s}\left(T_{1}\right) C_{s} \exp \left[\frac{E_{g}}{R_{g}}\left(\frac{1}{T_{1}}-\frac{1}{T_{2}}\right)\right]
$$

In above equation, $K_{s}\left(T_{1}\right)$ shows the dissolution rate constants at temperature $T_{1}, E_{g}$ is the activation energy and $R_{g}$ is the gas constant.

The acid diffusivity relation is given by Kalia and Glas-

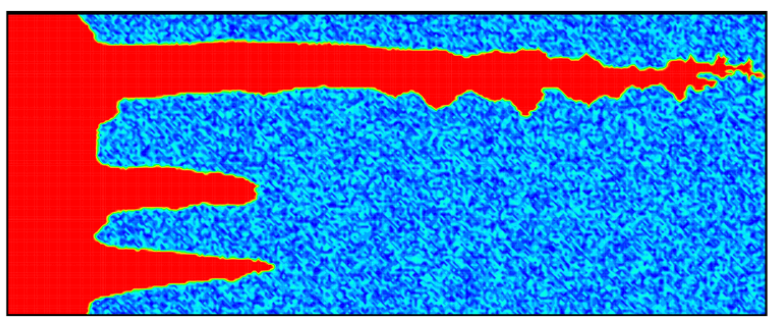

(b)

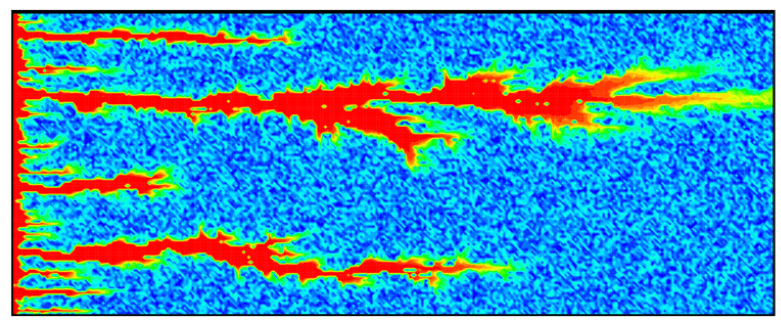

(d)

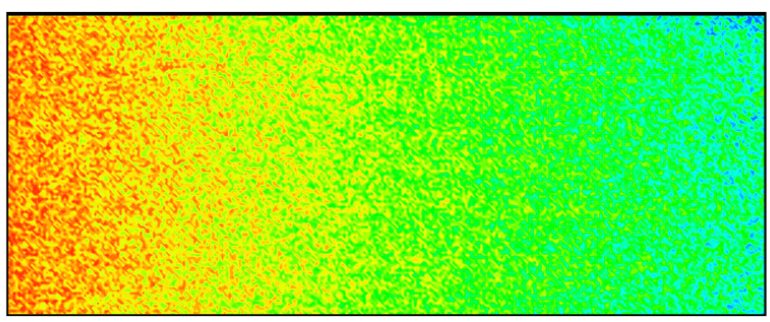

(f)

(e)

$\varepsilon$

$\begin{array}{lllllllllll}0.1 & 0.185 & 0.27 & 0.355 & 0.44 & 0.525 & 0.61 & 0.695 & 0.78 & 0.865 & 0.95\end{array}$

Fig. 3. Linear dissolution patterns for (a) $D a=5000$; (b) $D a=1000$; (c) $D a=500$; (d) $D a=100$; (e) $D a=10$; (f) $D a=1$. 
bergen (2009) as:

$$
\frac{D_{m 2}}{D_{m 1}}=\frac{\mu_{2}}{\mu_{1}} \frac{T_{2}}{T_{1}}
$$

where $D_{m 1}$ and $D_{m 2}$ are acid diffusivity values at different temperatures $T_{1}$ and $T_{2}$, and $\mu_{1}$ and $\mu_{2}$ are the acid viscosity values at $T_{1}$ and $T_{2}$ respectively.

\subsubsection{PVBT curves at different temperatures}

The simulation was run for regular $\mathrm{HCl}$ at 100 and 185 ${ }^{\circ} \mathrm{F}$. Fig. 4 displays the PVBT of acid versus the injection rate in a radial system. It can be seen that the essential PV is a function of the injection rate. By increasing the temperature, the rock dissolution at the inlet will increase due to a higher reaction rate. To reach a breakthrough and deeper penetration of acid, the optimum injection rate is directly proportional to the temperature. The same results were reported by Akanni (2015).

As displayed in Fig. 4, both PVBT and the optimal injection rate change with the rise of temperature. The significant alteration is mostly because of variations occurring in the reaction and transport mechanism. Balance among reaction, dispersion, and convection is achieved as the acid is injected at the optimum injection rate. By increasing the temperature, the acid diffusivity and reactivity will be increased. Therefore, a higher injection rate requires to return balance in the system and to create the dominant wormholes.

As shown in Fig. 4, at high injection rates, the high temperatures increase the convection and reaction, which leads to more efficient dissolution. Hence, a less significant volume of acid is required at high temperatures. However, at low injection rates, by increasing the temperature, as diffusion coefficient increases and more acid diffuses to the walls of the wormhole and is consumed there, the volumes of required acid to breakthrough increase. Also, at the optimum injection rate region, fewer amounts of $\mathrm{H}^{+}$can reach the wormhole tip as they are consumed at the inlet. Therefore, more acids are required to be injected to breakthrough the damage zone. In

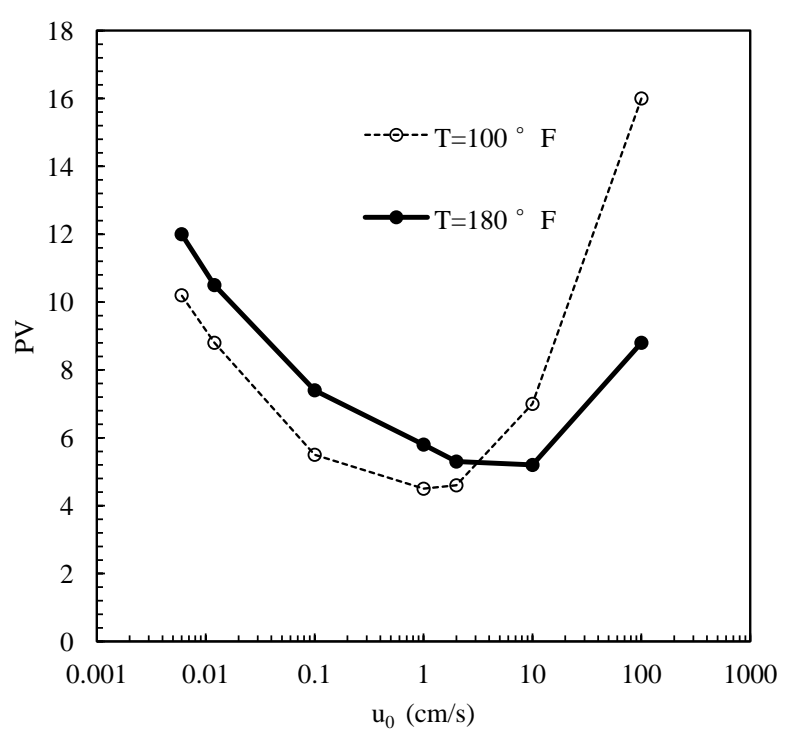

Fig. 4. Breakthrough curves for regular $\mathrm{HCl}$ at 100 and $185^{\circ} \mathrm{F}$.

some high-temperature treatments, $\mathrm{HCl}$ does not provide adequate stimulation results due to shortage of penetration or surface reactions. As the optimal pumping rate would be more than fracture gradient of the formation of carbonate reservoirs, using $\mathrm{HCl}$ for matrix acidizing cannot be successful.

\subsubsection{Dissolution pattern of regular $\mathrm{HCl}$ at different temperatures}

Fig. 5 shows dissolution pattern and wormhole propagation at low injection rate of $0.1 \mathrm{~cm} / \mathrm{s}$, for regular $\mathrm{HCl}$, at 100 and $185^{\circ} \mathrm{F}$. It is clear that face dissolution is increased by raising the temperature, which prevents deeper penetration of acid into the reservoir. Furthermore, by increasing the temperature, acid is spent on the walls of the wormhole instead of being transferred to the tip of the wormhole, and results in more acid usage and inefficient wormhole propagation (branched wormholes). $\mathrm{HCl}$ has high reaction and corrosion rates at high-

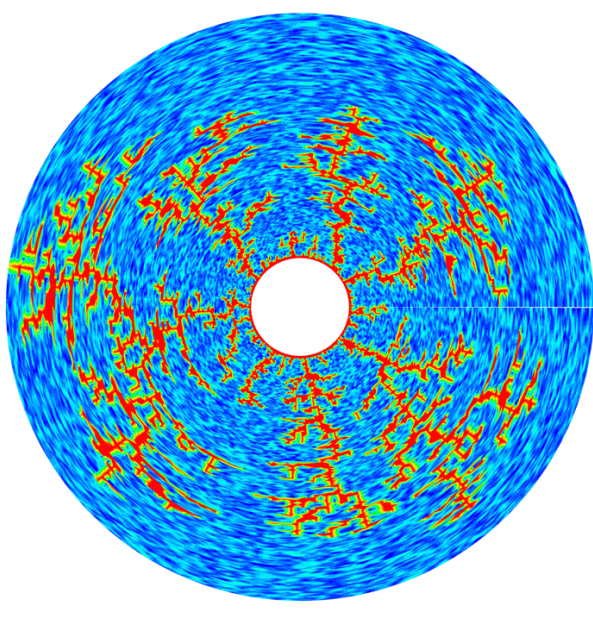

(a)
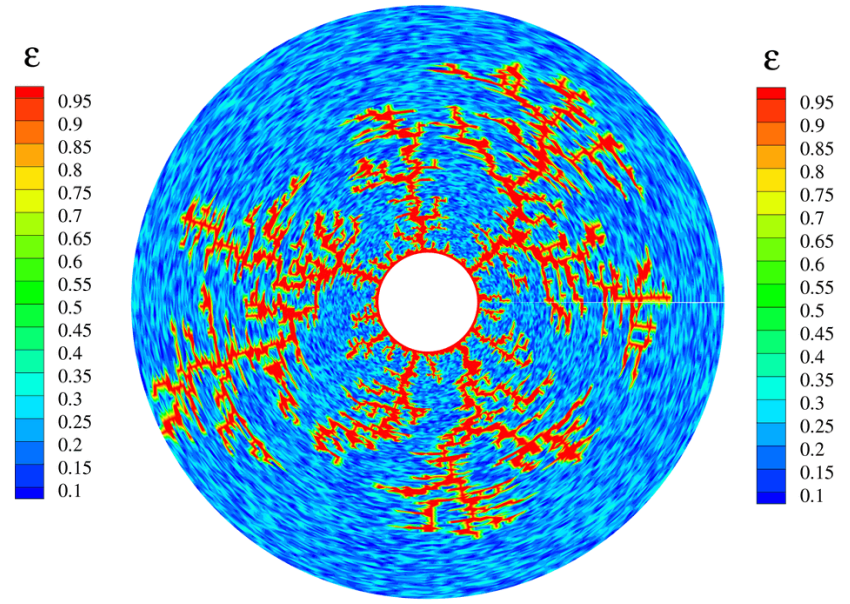

(b)

Fig. 5. Dissolution pattern of regular $\mathrm{HCl}$ at fixed injection rate $\left(u_{0}=0.1 \mathrm{~cm} / \mathrm{s}\right)$ with different temperature (a) $\mathrm{T}=100{ }^{\circ} \mathrm{F},(\mathrm{b}) \mathrm{T}=185{ }^{\circ} \mathrm{F}$. 
temperature wells. An acidizing system which is comparable to $\mathrm{HCl}$ in respects of price and availability is needed for these types of wells.

\subsection{Effect of type of acid}

Todays, due to the availability, high dissolution strengths, and the low cost of $\mathrm{HCl}$, most of the acid matrix operations of the carbonate reservoirs are carried out by this acid. However, there are limitations associated with $\mathrm{HCl}$ due to its aggressive nature such as high reaction rate. The reaction rate of $\mathrm{HCl}$ with carbonate is mass transfer limited so the rate of acid consumption is a function of the rate of acid injection. Matrix acidizing treatments at low injection rates causes the acid to be consumed prior to penetration into the reservoir in the area around the well. Moreover, operations with high injection rates are sometimes not possible due to the pressure limitations such as low fracture pressure. One of the alternative options in a high-temperature reservoir is in-situ generated $\mathrm{HCl}$ to behave like a Newtonian fluid and overcome high corrosion rates and fast reaction rate and of regular $\mathrm{HCl}$.

\subsubsection{Kinetic variables of the in-situ generated acid vs. 15 wt\% regular $\mathrm{HCl}$ acid}

Dissolution experiments by employing a rotating disk apparatus (RDA) for in-situ generated $\mathrm{HCl}$ was carried out by Arslan et al. (2017). The list of additives and their concentrations at which they used throughout their experiments are illustrated in Table 2. Furthermore, Table 3 shows measured viscosity, density and $\mathrm{pH}$ values of both acids.

The diffusion coefficient, reaction rate constant and kinematic viscosity at three different temperatures $(100,150$, $200{ }^{\circ} \mathrm{F}$ ) for the in-situ generated acid were determined and compared to that of $15 \mathrm{wt} \%$ regular $\mathrm{HCl}$ by Arslan et al. (2017). Referring to their experimental results, as it is visible

Table 2. Concentrations of additives used by Arslan et al. (2017).

\begin{tabular}{ll}
\hline Additive & Concentration, gpt \\
\hline Iron Control Agent & 8 \\
Non-Emulsifier & 4 \\
Corrosion inhibitor & 6 \\
Corrosion Inhibitor Intensifier & 40 \\
\hline
\end{tabular}

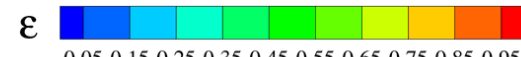

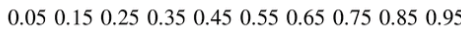

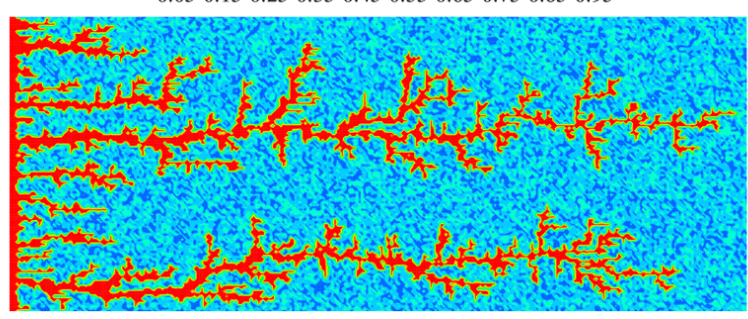

(a)
Table 3. Density, viscosity and $\mathrm{pH}$ values of acids measured at $75^{\circ} \mathrm{F}$ (Arslan et al. 2017)

\begin{tabular}{llll}
\hline Acid type & Density, g/cm & Viscosity, $\mathrm{cp}$ & $\mathrm{pH}$ \\
\hline HCl $15 \%+$ additives & 1.082 & 1.30 & 0 \\
$\begin{array}{l}\text { In-situ generated acid } \\
+ \text { additives }\end{array}$ & 1.14 & 1.92 & 0 \\
\hline
\end{tabular}

in Table 4, reaction rate constant and diffusion coefficient of in-situ generated $\mathrm{HCl}$ are much lower than $15 \mathrm{wt} \% \mathrm{HCl}$ at $100{ }^{\circ} \mathrm{F}$. Moreover, they noted $15 \mathrm{wt} \% \mathrm{HCl}$ at 100 is nearly 4 times more diffusive than in-situ generated $\mathrm{HCl}$ at $100{ }^{\circ} \mathrm{F}$.

\subsubsection{Dissolution pattern for two types of acid at the same temperatures}

The application of an in-situ generated $\mathrm{HCl}$ acid system by Sokhanvarian et al. (2017) and the results obtained are encouraging to consider this acid system for carbonate matrix acidizing at high temperatures. Because of the lower reaction rate and diffusivity, in-situ generated $\mathrm{HCl}$ is more effective in terms of wormhole propagation (Sokhanvarian et al., 2017). This results in the less required PVBT of acid when compared to regular $\mathrm{HCl}$ under the same conditions. In this study, a comparison between regular $\mathrm{HCl}$ and in-situ generated $\mathrm{HCl}$ is conducted through a two-dimensional linear model. The simulation was performed at $100{ }^{\circ} \mathrm{F}$ for both $\mathrm{HCl} 15 \%$ and in-situ generated $\mathrm{HCl}$ based on a fixed injection rate. The wormhole propagation from the simulation is shown in Fig. 6 . As can be seen, more face dissolution is associated with using regular $\mathrm{HCl}$ compared to in-situ generated $\mathrm{HCl}$, which leads to shallower penetration of acid. The formed wormholes are more branched in the case of regular $\mathrm{HCl}$ that causes regular $\mathrm{HCl}$ to be spent more on the wormhole walls rather than being transferred toward the tip of the wormhole. On the other hand, less face dissolution can be seen when in-situ generated $\mathrm{HCl}$ is used that results in more efficient wormhole propagation with a less injected PV of acid.

The dissolution pattern for both acids at $100{ }^{\circ} \mathrm{F}$ and 0.01 $\mathrm{cm} / \mathrm{s}$ is presented in Figs. 7(a) and 7(b). Face dissolution near the wellbore and wormholes with larger diameter can be seen if regular $\mathrm{HCl}$ is used (Fig. 7(b)). As reported in Table 4, the in-situ generated $\mathrm{HCl}$ is more viscous than the regular acid.

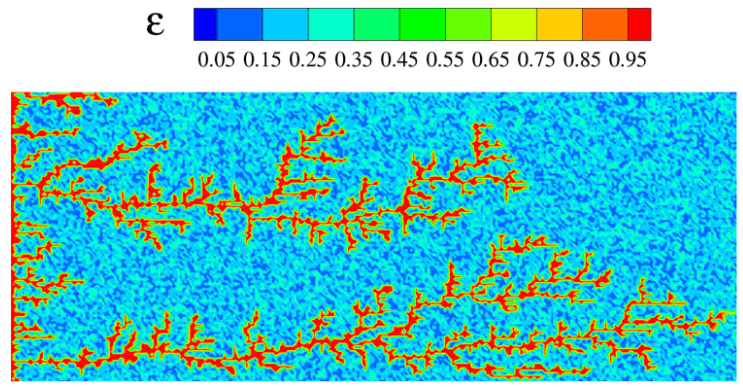

(b)

Fig. 6. Dissolution pattern for both acid systems at a fixed injection rate $\left(u_{0}=0.01 \mathrm{~cm} / \mathrm{s}\right)$ and $100{ }^{\circ} \mathrm{F}$. (a) $\mathrm{HCl} 15 \%$; (b) in-situ generated $\mathrm{HCl}$. 


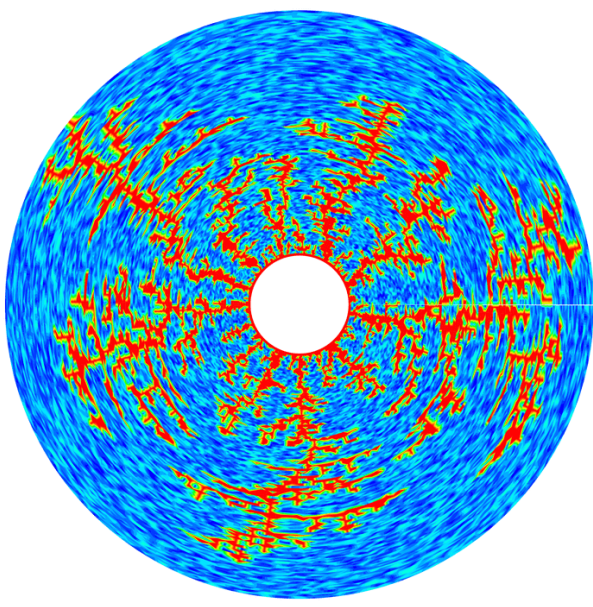

(a)

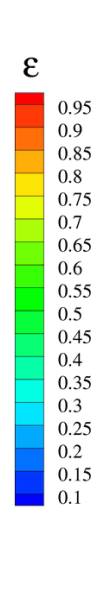

1

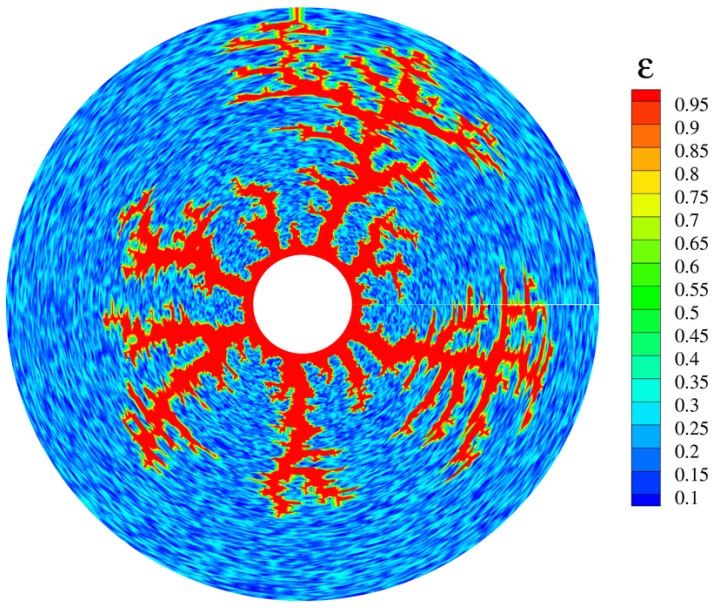

(b)

Fig. 7. Dissolution pattern for both acid systems in radial flow at a fixed injection rate $\left(u_{0}=0.01 \mathrm{~cm} / \mathrm{s}\right)$ and $100{ }^{\circ} \mathrm{F}$. (a) in-situ generated $\mathrm{HCl}$; (b) $\mathrm{HCl} 15 \%$.

Table 4. Kinematic parameters calculated at three different temperatures for in-situ generated $\mathrm{HCl}$ and at $100{ }^{\circ} \mathrm{F}$ for $15 \mathrm{wt} \% \mathrm{HCl}(\mathrm{Arslan}$ et al., 2017 ).

\begin{tabular}{lllll}
\hline Acid type & $\begin{array}{l}\text { Temperature, } \\
{ }^{\circ} \mathrm{F}\end{array}$ & $\begin{array}{l}\text { Reaction rate constant, } \\
\mathrm{gmole} /\left(\mathrm{s} \cdot \mathrm{cm}^{2}\right) \cdot(\mathrm{rad} / \mathrm{s})^{0.5}\end{array}$ & $\begin{array}{l}\text { Kinematic viscosity, } \\
\mathrm{cm}^{2} / \mathrm{s}\end{array}$ & $\begin{array}{l}\text { Diffusion coefficient, } \\
\mathrm{cm}^{2} / \mathrm{s}\end{array}$ \\
\hline $15 \mathrm{wt} \% \mathrm{HCl}$ & 100 & $7.26 \mathrm{e}-6$ & 0.00836 & $3.13 \mathrm{e}-5$ \\
In-situ generated $\mathrm{HCl}$ & 200 & $1.66 \mathrm{e}-5$ & 0.00567 & $8.97 \mathrm{e}-5$ \\
In-situ generated $\mathrm{HCl}$ & 150 & $6.99 \mathrm{e}-6$ & 0.00758 & $2.51 \mathrm{e}-5$ \\
In-situ generated $\mathrm{HCl}$ & 100 & $2.93 \mathrm{e}-6$ & 0.01142 & $7.31 \mathrm{e}-6$ \\
\hline
\end{tabular}

Higher viscosity reduces the mobility of the acid to the walls of the wormhole and the acid will have a chance to get to the tip of the wormhole. This eventually results in a channel with a smaller diameter and a more efficient structure.

\subsubsection{Comparison of PVBT for two types of acid}

Fig. 8 displays the injected PV of acid versus the injection rate of acid. The PVBT of acid especially at lower injection rates is decreased in the case of in-situ generated $\mathrm{HCl}$. This is due to the lower reaction rate and diffusivity of in-situ generated $\mathrm{HCl}$ compared to regular $\mathrm{HCl}$. The lower reaction rate of in-situ generated $\mathrm{HCl}$ provides an opportunity for the acid to penetrate deeper into the reservoir, which leads to less PVBT of acid. These simulation results also show that the optimum injection rate is decreased due to slower mass transfer from the bulk to the rock-acid interface, so a lower injection rate is needed to breakthrough if in-situ generated $\mathrm{HCl}$ is used (Fig. 8).

\section{Conclusions}

In this study, we employed a TSC model to examine the phenomenon of wormhole formation and propagation. The effect of temperature on the acid breakthrough curve and dissolution pattern was investigated for regular $\mathrm{HCl}$ acid and the results compared with the newly developed acid system, called in-situ generated $\mathrm{HCl}$. From the numerical simulation, the following conclusions could be drawn:

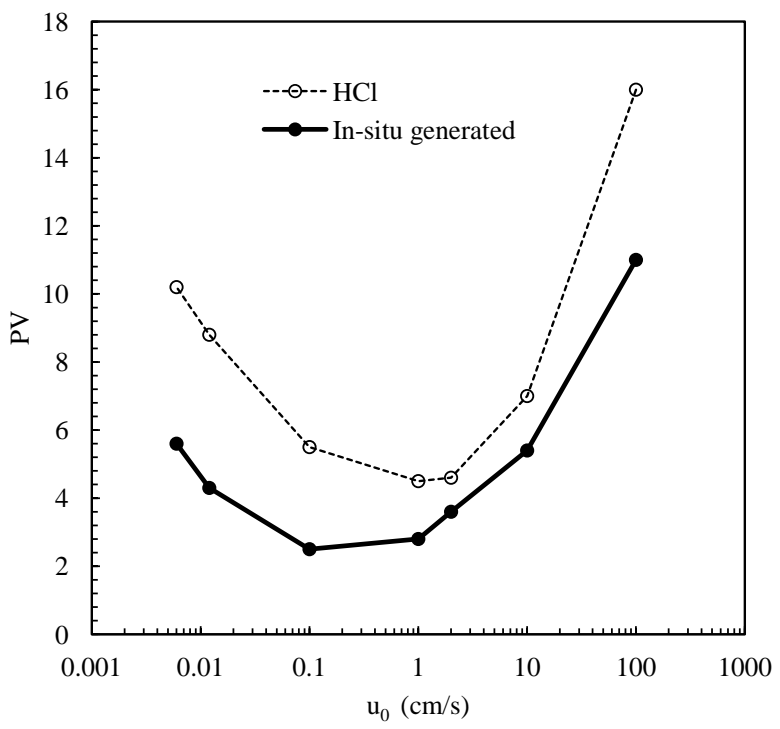

Fig. 8. Comparison of breakthrough curves of $15 \mathrm{wt} \%$ regular $\mathrm{HCl}$ and in-situ generated $\mathrm{HCl}$ at $100{ }^{\circ} \mathrm{F}$.

1) Results of numerical simulation showed that the PVBT of acid especially at lower injection rates can be decreased in the case of in-situ generated $\mathrm{HCl}$.

2) Simulation results showed that a lower injection rate is needed to breakthrough if in-situ generated $\mathrm{HCl}$ is used.

3) Based on created dissolution patterns,in-situ generated $\mathrm{HCl}$ not only decreases face dissolution but also increases 
the acid penetration into the reservoir. This leads to an increase in the efficiency of matrix acidizing treatment.

4) These results display that the in-situ generated $\mathrm{HCl}$ could be considered as a suitable alternative to $\mathrm{HCl}$ especially when due to low injectivity of a reservoir, low injection rates is required during matrix acidizing of carbonates.

\section{Abbreviations}

\section{Symbols}

$U=$ velocity, $\mathrm{cm} / \mathrm{s}$

$K=$ permeability tensor

$P=$ pressure, atm

$\mu=$ acid viscosity, $\mathrm{cp}$

$\varepsilon=$ porosity of the medium

$t=$ time, $\mathrm{s}$

$C_{s}=$ acid concentration in the fluid-solid interface, $\mathrm{gr} / \mathrm{cm}^{3}$

$C_{f}=$ acid concentration in fluid, $\mathrm{gr} / \mathrm{cm}^{3}$

$D_{e}=$ effective dispersion tensors

$K_{c}=$ local mass transfer coefficient, $\mathrm{cm} / \mathrm{s}$

$a_{v}=$ interfacial area, $\mathrm{cm}^{2}$

$R\left(C_{S}\right)=$ reaction kinetics, $\mathrm{gr} /\left(\mathrm{cm}^{2} \cdot \mathrm{s}\right)$

$\alpha=$ acid dissolving power

$\rho_{s}=$ rock density, $\mathrm{gr} / \mathrm{cm}^{3}$

$K_{s}=$ surface-dissolution reaction-rate constant, $\mathrm{cm} / \mathrm{s}$

$H=$ height of the area, $\mathrm{cm}$

$L=$ characteristic length in the flow direction, $\mathrm{cm}$

$u^{*}=$ dimensionless velocity in $\mathrm{x}$-direction

$v^{*}=$ dimensionless velocity in $\mathrm{y}$-direction

$t^{*}=$ dimensionless time

$u=$ velocity in $\mathrm{x}$-direction, $\mathrm{cm} / \mathrm{s}$

$v=$ velocity in $\mathrm{y}$-direction, $\mathrm{cm} / \mathrm{s}$

$r=$ dimensionless pore radius

$r_{0}=$ initial mean pore radius, $\mathrm{cm}$

$r_{p}=$ average pore radius, $\mathrm{cm}$

$u_{0}=$ inlet velocity, $\mathrm{cm} / \mathrm{s}$

$a_{0}=$ initial interface area, $\mathrm{cm}^{2}$

$A_{v}=$ dimensionless interfacial area

$k_{0}=$ initial permeability, md

$k^{*}=$ dimensionless permeability

$k=$ permeability, md

$C_{0}=$ inlet concentration, $\mathrm{gr} / \mathrm{cm}^{3}$

$c_{f}=$ dimensionless acid concentration in fluid

$c_{s}=$ dimensionless acid concentration in the fluid-solid interface

$P_{e}=$ outlet pressure, atm

$\varphi^{2}=$ pore scale Thiele modulus (notation of this parameter existed in paper)

$D_{m}=$ molecular diffusivity of the acid, $\mathrm{cm}^{2} / \mathrm{s}$

$\alpha_{0}=$ aspect ratio

$D a=$ Damkohler number

$N_{a c}=$ acid capacity number

$P e_{L}=$ the axial Peclet number

$\Phi^{2}=$ macroscopic Thiele modulus (notation of this parameter existed in paper)

$p=$ pressure, atm

$S h=$ Sherwood number

$\alpha_{O S}=$ constant in dispersion correlation $\lambda_{x}=$ constant in axial-dispersion correlation

$\lambda_{T}=$ constant in transverse-dispersion correlation

$\varepsilon_{0}=$ average porosity

$\beta=$ pore-structure-relation constant

$S h_{\infty}=$ asymptotic Sherwood number

$R_{e}=$ Reynolds number

$S c=$ schmidt number

$D_{e x}=$ longitudinal-dispersion coefficient, $\mathrm{cm}^{2} / \mathrm{s}$

$D_{e T}=$ transverse-dispersion coefficient, $\mathrm{cm}^{2} / \mathrm{s}$

$\xi=$ varies randomly from $-\Delta \varepsilon$ to $\Delta \varepsilon$

$\Delta \varepsilon_{0}=$ porosity-heterogeneity magnitude

$K_{s}\left(T_{1}\right)=$ shows the dissolution rate constants at constants at temperature $T_{1}$

$E_{g}=$ activation energy

$R_{g}=$ gas constant

$v=$ kinematic viscosity, $\mathrm{cm}^{2} / \mathrm{s}$

\section{Nomenclature}

$\mathrm{HCl}=$ hydrochloric

PVBT $=$ pore volumes to breakthrough

TSM = two-scale model

$\mathrm{PV}=$ pore volume

$\mathrm{RDA}=$ rotating disk apparatus

$\mathrm{TSC}=$ two-scale continuous

\section{Acknowledgement}

The authors would like to thank the reviewers for all of their careful, constructive and insightful comments in relation to this work.

\section{Conflict of interest}

The authors declare no competing interest.

Open Access This article is distributed under the terms and conditions of the Creative Commons Attribution (CC BY-NC-ND) license, which permits unrestricted use, distribution, and reproduction in any medium, provided the original work is properly cited.

\section{References}

Akanni, O. O. Mathematical modeling of wormhole propagation during matrix acidizing of carbonate reservoir. Texas, Texas A\&M University, 2015.

Akanni, O. O., Nasr-El-Din, H. A. The accuracy of carbonate matrix-acidizing models in predicting optimum injection and wormhole propagation rates. Paper SPE 172575 Presented at SPE Middle East Oil \& Gas Show and Conference, Manama, Bahrain, 8-11 March, 2015.

Aldakkan, B., Ahmed, M., Gomaa, J., et al. Low viscosity retarded acid system: A novel alternative to emulsified acids. Paper SPE 192175 Presented at SPE Kingdom of Saudi Arabia Annual Technical Symposium and Exhibition, Dammam, Saudi Arabia, 23-26 April, 2018.

Ali, M. T., Nasr-El-Din, H. A. A robust model to simulate dolomite-matrix acidizing. SPE Production \& Operations, 2019, 34(1): 109-129.

Ali, M. T., Nasr-El-Din, H. A. New insights into carbonate matrix acidizing treatments a mathematical and experimental study. SPE Journal, 2020, 25(3): 1272-1284. 
Al-Mutairi, S. H., Hill, A. D., Nasr-El-Din, H. A. Effect of droplet size, emulsifier concentration and acid volume fraction on the rheological properties and stability of emulsified acids. Paper SPE 107741 Presented at European Formation Damage Conference, Scheveningen, The Netherlands, 30 May-1 June, 2007.

Al-Mutairi, S. H., Nasr-El-Din, H. A., Hill, A. D. Effect of droplet size on the reaction kinetics of emulsified acid with calcite. SPE Journal, 2009, 14(4): 606-616.

Arslan, E., Sokhanvarian, K., Nasr-El-Din, H. A. Reaction rate of a novel in-situ generated $\mathrm{HCl}$ acid and calcite. Paper SPE 187059 Presented at SPE Annual Technical Conference and Exhibition, San Antonia, Texas, USA, 9-11 October, 2017.

Cairns, A. J., Al-Muntasheri, G. A., Sayed, M. Targeting enhanced production through deep carbonate stimulation: Stabilized acid emulsions. Paper SPE 178967 Presented at SPE International Conference and Exhibition on Formation Damage Control, Lafayette, Louisiana, USA, 24-26 February, 2016.

Chang, F., Nasr-El-Din, H. A. Matrix acidizing of carbonate reservoirs using organic acids and mixture of $\mathrm{HCl}$ and organic acids. Paper SPE 116601 Presented at SPE Annual Technical Conference and Exhibition, Denver, Colorado, USA, 21-24 September, 2008.

Cohen, C. E., Ding, D., Quintard, M., et al. From pore scale to wellbore scale: Impact of geometry on wormhole growth in carbonate acidization. Chemical Engineering Science, 2007, 63(12): 3088-3099.

Danckwerts, P. V. Continuous flow systems: Distribution of residence times. Chemical Engineering Science, 1953, 2(1): 1-13.

Fredd, C. N., Fogler, H. S. Influence of transport and reaction on wormhole formation in porous media. AIChE Journal, 1998, 44(9): 1933-1949.

Fredd, C. N. Dynamic model of wormhole formation demonstrates conditions for effective skin reduction during carbonate matrix acidizing. Paper SPE 59537 Presented at SPE Permian Basin Oil and Gas Recovery Conference, Midland, Texas, USA, 21-23 March, 2000.

Glasbergen, G., Kalia, N., Talbot, M. S. The optimum injection rate for wormhole propagation: myth or reality? Paper SPE 121464 Presented at the 8th European Formation Damage Conference, Scheveningen, The Netherlands, 27 May, 2009.

Golfier, F., Zarcone, C., Bazin, B. On the ability of a Darcyscale model to capture wormhole formation during the dissolution of a porous medium. Journal of Fluid Mechanics, 2002, 457: 213-254.

Kalia, N., Balakotaiah, V. Effect of medium heterogeneities on the reactive dissolution of carbonates. Chemical Engineering Science, 2009, 64(2): 2376-390.

Kalia, N., Glasbergen, G. Wormhole formation in carbonates under varying temperature conditions. Paper SPE 121803 Presented at the 8th European Formation Damage Conference, Scheveningen, The Netherlands, 27-29, May, 2009.

Kankaria, S., Nasr-El-Din, H. A., Rimassa, S. Matrix acidizing of carbonate rocks using new mixtures of $\mathrm{HCl} /$ methanesulfonic acid. Paper SPE 184528 Presented at SPE International Conference on Oilfield Chemistry, Montgomery, Texas, USA, 3-5 April, 2017.

Liu, P., Couples, G. D., Yao, J. A general method for simulating reactive dissolution in carbonate rocks with arbitrary geometry. Computational Geosciences, 2018, 22(5): 1187-201.

Liu, X., Ormond, A., Bartko, K. A geochemical reactiontransport simulator for matrix acidizing analysis and design. Journal of Petroleum Science and Engineering, 1997, 17(1-2): 181-196.

Mahmoud, M., Nasr-El-Din, H. A. Challenges during shallow and deep carbonate reservoirs stimulation. Journal of Energy Resources Technology, 2015, 137(1): 012902.

Mason, T. G., Bibette, J., Weitz, D. A. Yielding and flow of monodisperse emulsions. Journal of Colloid and Interface Science, 1996, 179(2): 439-448.

McDuff, D., Shuchart, C. E., Jackson, S. Understanding wormholes in carbonates unprecedented experimental scale and 3-D visualization. Paper SPE 134379 Presented at SPE Annual Technical Conference and Exhibition, Florence, Italy, 19-22 September, 2010.

Nasr-El-Din, H. A., Dana, H., Tomos, V. Field Treatment to stimulate an oil well in an offshore sandstone reservoir using a novel, low-corrosive, environmentally friendly fluid. Journal of Canadian Petroleum Technology, 2015, 54(5): 289-297.

Panga, M. K., Ziauddin, M., Balakotaiah, V. A new model for predicting wormhole structure and formation in acid stimulation of carbonates. Paper SPE 86517 Presented at SPE International Symposium and Exhibition on Formation Damage Control, Lafayette, Louisiana, 1820 February, 2004.

Panga, M. K., Ziauddin, M., Balakotaiah, V. Two-scale continuum model for simulation of wormholes in carbonate acidization. AIChE Journal, 2005, 51(12): 3231-3248.

Shuchart, C. E., Gdanski, R. D. Improved success in acid stimulations with a new organic HF system. Paper SPE 36907 Presented at European Petroleum Conference, Milan, Italy, 22-24 October, 1996.

Sokhanvarian, K., Nasr-El-Din, H. A., de Wolf, C. A. Thermal stability of oilfield aminopolycrboxylic acids/salts. SPE Production \& Operations, 2016, 31 (1): 12-21.

Sokhanvarian, K., Pummarapanthu, T., Arslan, E. A new insitu generated acid system for carbonate dissolution in sandstone and carbonate reservoirs. Paper SPE 184506 Presented at SPE International Conference on Oilfield Chemistry, Montgomery, Texas, USA, 3-5 April, 2017.

Yuan, T., Guan, Q. Numerical investigation of wormhole formation during matrix acidizing of carbonate rocks by coupling stokes-brinkman equation with reactive transport model under radial flow conditions. Paper SPE 199262 Presented at SPE International Conference and Exhibition on Formation Damage Control, Lafayette, Louisiana, USA, 19-21 February, 2020. 